\title{
Quantification of total fatty acids in microalgae: comparison of extraction and transesterification methods
}

\author{
Lillie R. Cavonius • Nils-Gunnar Carlsson • \\ Ingrid Undeland
}

Received: 10 April 2014 /Revised: 22 August 2014 / Accepted: 2 September 2014 / Published online: 16 September 2014

(C) The Author(s) 2014. This article is published with open access at Springerlink.com

\begin{abstract}
Determination of microalgaes' fatty acid content is often done with chloroform and methanol according to the Bligh and Dyer extraction, though faster methods exist. A number of comparisons between the Bligh and Dyer and faster methods have resulted in contradicting data, possibly due to differences in algae used and the different versions of the Bligh and Dyer method applied. Here, various forms of directtransesterification (D-TE) and two-step transesterification (2$\mathrm{TE}$ ), including three versions developed in our lab, are compared with the original Bligh and Dyer (Can J Biochem Physiol 37: 911-917, 1959) extraction and two modifications thereof (Lee et al. J AOAC Int 79:487-492, 1996, and our own acidified version) on microalgae with different cell walls: Isochrysis galbana, Nannochloropsis oculata, and Phaeodactylum tricornutum. In total, fatty acid extracts from 11 methods were separated and quantified by gas chromatography with mass spectrometry. Results show that, for $N$. oculata and P. tricornutum, methods based on chloroform-methanol underestimated the fatty acid content compared with the 2-TE and D-TE methods, which gave similar results. Moreover, DTE methods are faster than chloroform-methanol methods and use chemicals that are less toxic. Of the D-TE methods, the ones using hydrochloric acid and sulfuric acid recovered the most fatty acids, while boron trifluoride recovered slightly less. The main qualitative difference between the fatty acids recovered was that the chloroform-methanol methods recovered less saturated fatty acids in P. tricornutum.
\end{abstract}

Electronic supplementary material The online version of this article (doi:10.1007/s00216-014-8155-3) contains supplementary material, which is available to authorized users.

L. R. Cavonius $(\bowtie) \cdot$ N.-G. Carlsson $\cdot$ I. Undeland Food Science, Division of Life Science, Department of Chemical and Biological Engineering, Chalmers University of Technology,

41296 Gothenburg, Sweden

e-mail: 1illie@chalmers.se
Keywords Microalgae $\cdot$ Fatty acids $\cdot$ Bligh and Dyer extraction $\cdot$ Direct transesterification

$\begin{array}{ll}\text { Abbreviations } \\ \text { 2-TE } & \text { Two-step transesterification } \\ \text { BHT } & \begin{array}{l}\text { Butylated hydroxytoluene [systematic name: } \\ \text { 2,6-bis(1,1-dimethylethyl)-4-methylphenol] }\end{array} \\ \text { D-TE } & \text { Direct transesterification } \\ \text { FAME } & \text { Fatty-acid methyl-ester } \\ \text { MUFA } & \text { Monounsaturated fatty acid } \\ \text { PUFA } & \text { Polyunsaturated fatty acid } \\ \text { SFA } & \text { Saturated fatty acid }\end{array}$

\section{Introduction}

Microalgae are currently being investigated as possible sources of nutrition and biodiesel. In both applications, the main focus lies on the fatty acids produced by the microalgae. Therefore, methods for determining both the total quantity and type of the fatty acids in microalgae are needed. At present, there are many available methods, though there are only limited comparisons between them.

In 1959, Bligh and Dyer developed an extraction method based on chloroform and methanol, a solvent combination which proved to have a good ability to penetrate cells and recover total lipids from fish tissue [1]. The method is widely popular (currently cited over 31,000 times) and therefore allows for comparison of results from different studies, comprising many different matrices. However, it is important to stress that there are numerous modifications of the Bligh and Dyer method, some being presented as method modifications, others still being referred to as "Bligh and Dyer". Although the Bligh and Dyer extraction is widely applied to microalgae, chloroform-methanol may not be the most appropriate 
solvent system for lipid extraction, due to differences in cell wall and lipid composition [2-18]. Furthermore, the Bligh and Dyer method was originally described as particularly good for tissue samples with low lipid content [1]. Later, Lee et al. demonstrated that increasing the chloroform-to-methanol ratio was a way to get accurate results for fish with $>6 \%$ lipid content [19]. Microalgae considered for biotech applications usually contain at least $10 \%$ lipids. An overview of the Bligh and Dyer method's main steps is shown in Fig. 1.

Two-step transesterification (2-TE) is a method in which biomass is treated with strong alkali, and fatty acids are subsequently methylated with an acidic catalyst. The alkaline condition breaks ester bonds, liberates the fatty acids [20], and possibly facilitates later extraction by degrading structures such as cell walls [21]. A method was developed in 1996 by which fatty acids were saponified and extracted from microalgae in a single step [22]. The resulting fatty acid extract can be further methylated and analyzed, as demonstrated by Burja et al. (2007) [23].

Direct-transesterification (D-TE) is a third method for analyzing fatty acids. Methods based on chloroform-methanol, as well as 2-TE methods require multiple steps before the fatty acids are methylated in the final step. D-TE applies the methylation agent directly to the biomass and thus reduces extraction steps. This technique was applied to microalgae already in 1990 [24]. Many different catalysts are currently used for D-TE, the most common being: hydrochloric acid $(\mathrm{HCl})$, boron trifluoride $\left(\mathrm{BF}_{3}\right)$, and sulfuric acid $\left(\mathrm{H}_{2} \mathrm{SO}_{4}\right)$. However, according to the literature, there does not appear to be any agreement on which, if any, gives the most accurate results [20, 25-27].

To the best of our knowledge, it was not until 2007 that Burja et al. compared chloroform-methanol extraction with 2TE and D-TE, specifically addressing microalgae [23]. The comparison was carried out on Thraustrochytrium sp., with the authors concluding that a miniaturized Bligh and Dyer gave the highest fatty acid yields [23]. Interestingly, Griffiths et al. compared various chloroform-methanol methods with a 2-TE on Chlorella vulgaris, Scenedesmus sp., and Nannochloropsis sp., concluding that the 2-TE method gave the highest yield and required less time and effort [28]. The conflicting results could be explained either by the different method versions used, or by differences in the algae's cell walls. Therefore, further comparisons of fatty acid quantifying principles using morphologically different algal species is warranted.

Here, the aim was to compare three main groups of fatty acid-recovering methods by using three species of microalgae with different types of cell walls: Phaeodactylum tricornutum, with an organic cell wall [29, 30]; Nannochloropsis oculata, with its robust algaenan cell wall [31, 32]; and the comparatively fragile Isochrysis galbana [33]. Outcomes considered were total fatty acid yield, fatty acid profile, and the general

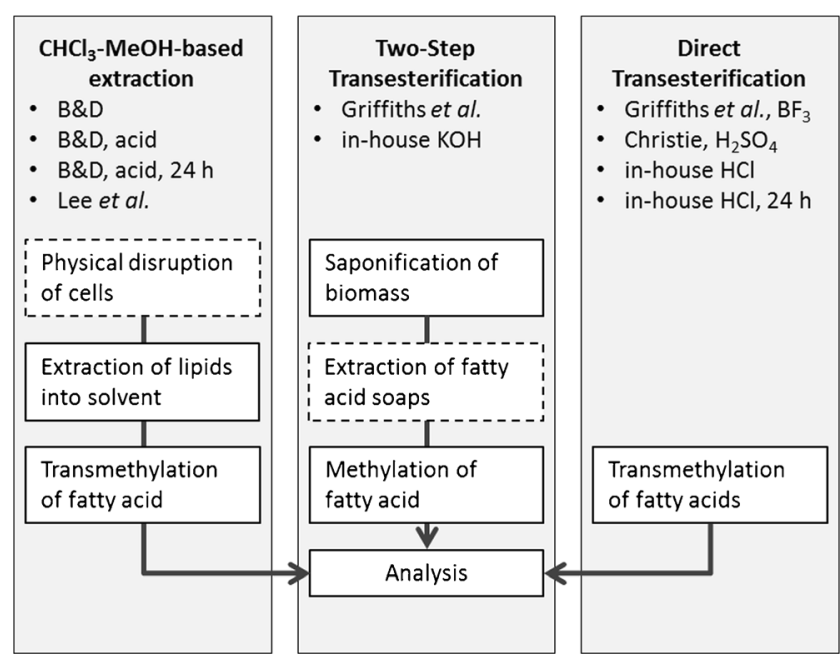

Fig. 1 Simplified flowchart of the workflow in fatty acid analysis in microalgae by chloroform-methanol-based extraction, two-step transesterification, and direct transesterification. $B \& D$ Bligh and Dyer. The dashed box indicates an optional step, not necessarily present in all versions of the method

practicality of the method. The three main principles for recovering fatty acids were: (1) chloroform-methanol-based extraction, (2) 2-TE and (3) D-TE. Within each main method group, several different versions were compared. For the Bligh and Dyer, this is justified by the many variations in circulation. 2-TE is known to give satisfactory results in previous studies and was included as a reference [23, 28]. We also present a new aggressive 2-TE method which was developed for disrupting and recovering fatty acids from algae with tough cells walls. Finally, different catalysts and versions of the D-TE were compared with find one that gives high yield, cuts down on toxic chemicals, and saves analysis time.

\section{Material and methods}

\section{General preparation of microalgae}

Microalgae were purchased dried from Necton (Olhão, Portugal, in 2012) and consisted of the following species: I. galbana, N. oculata, and P. tricornutum. It was confirmed that there was no water present (by freeze-drying). For all methods, $1 \mathrm{mg}$ of the fatty acid $\mathrm{C} 23: 0$ in chloroform was added as an internal standard to $12 \mathrm{~mL}$ glass tubes with Teflon screw-caps. Solvent was evaporated under nitrogen gas at $40{ }^{\circ} \mathrm{C}$ before $21 \pm 4 \mathrm{mg}$ of algal powder was weighed in. Since the areas of internal standard obtained in the chromatogram were in the same order of magnitude in all but one case, a second internal standard was not used. All fatty acidrecovering methods were carried out in triplicate. 
Chloroform-methanol extraction methods

\section{Bligh and Dyer's extraction}

The original Bligh and Dyer method [1] was followed as closely as possible, although several modifications were required, since it was scaled down by a factor of 1,000 . In brief, $20 \mathrm{mg}$ algae were wetted for $60 \mathrm{~min}$ with $80 \mu \mathrm{L}$ milli-Q water before $300 \mu \mathrm{L}$ of chloroform-methanol (1:2) was added. Samples were vortexed for $2 \mathrm{~min}$. Thereafter, $100 \mu \mathrm{L}$ of chloroform was added; tubes were vortexed for $30 \mathrm{~s}$; $100 \mu \mathrm{L}$ milli-Q water was added to create a two-phase system and vortexed for $30 \mathrm{~s}$ more. Tubes were centrifuged $(2,500 \times g$ for $6 \mathrm{~min}$ ), the clear aqueous phase discarded, the chloroform phase recovered, and the residue re-extracted with $100 \mu \mathrm{L}$ chloroform, centrifuging as above and pooling the recovered chloroform with the first portion. Chloroform extracts were methylated as described in "In-house methanolic- $\mathrm{HCl}$ transesterification" section and separated by gas chromatography-mass spectrometry (GC-MS) as described in "Analysis of fatty acids by GC-MS" section.

\section{Bligh and Dyer's acidic extraction}

The Bligh and Dyer method was followed as described above ("Bligh and Dyer's extraction" section), with our own modification where a two-phase system was created by adding $100 \mu \mathrm{L}$ of $0.1 \mathrm{M} \mathrm{HCl}$ instead of milli-Q water. This precaution was taken to ensure that the fatty acids were protonated, making it more likely for the fatty acids to be present in the organic phase. The $\mathrm{pH}$ of the initial biomass was measured by suspending $20 \mathrm{mg}$ of each type of algae in $200 \mathrm{~mL}$ milli-Q water; after sedimentation, ca. $10 \mu \mathrm{L}$ of supernatant was spotted onto indicator paper (Macherey-Nagel Tri-test, Germany). Likewise, the $\mathrm{pH}$ of the aqueous phase remaining at the end of the extraction was measured by spotting $10 \mu \mathrm{L}$ onto indicator paper. The organic phase was methylated as described in "In-house methanolic- $\mathrm{HCl}$ transesterification" section and separated as described in "Analysis of fatty acids by GC-MS" section.

\section{Bligh and Dyer's acidic extraction with overnight incubation}

The Bligh and Dyer acidic method was followed as described above ("Bligh and Dyer's acidic extraction" section), with the exception that the initial extraction with chloroform and methanol was extended to $24 \mathrm{~h}$ by incubating samples in the dark at room temperature on an orbital shaker running at $300 \mathrm{rpm}$. The rational for increasing the extraction time was to check if more fatty acids could be recovered, compared with the regular extraction time. The organic phase was methylated as described in "In-house methanolic-HCl transesterification" section and separated as described in "Analysis of fatty acids by GC-MS" section.

\section{Lee et al.'s extraction}

This method is a further development of the one published by Lee et al. [19]: To the microalgae, $8.0 \mathrm{~mL}$ of chloroformmethanol (2:1) was added, containing $0.05 \%(w / v)$ of the antioxidant butylated hydroxytoluene (BHT; Fluka, Sweden). Tubes were vortexed for $60 \mathrm{~s}$ before adding $3.0 \mathrm{~mL} 0.5 \%$ $(w / v)$ sodium chloride solution. Tubes were then vortexed $15 \mathrm{~s}$ and centrifuged $(2,000 \times g$ for $6 \mathrm{~min})$. The organic phase was transferred to a new tube, methylated as described in "Inhouse methanolic- $\mathrm{HCl}$ transesterification" section and separated as described in "Analysis of fatty acids by GC-MS" section.

Two-step transesterification (2-TE) methods

\section{Griffiths et al.'s transesterification}

This method was first published by Giffiths et al. [28], with minor changes applied here. Since dried microalgae were used, a water scavenger was not added here. Methanolic sodium methoxide, 0.5 M (Sigma-Aldrich, Sweden), $1.0 \mathrm{~mL}$, was added, and the tubes were incubated at $80{ }^{\circ} \mathrm{C}$ for $20 \mathrm{~min}$ at $200 \mathrm{rpm}$ shaking (the original method calls for $300 \mathrm{rpm}$, faster than our equipment could run). After allowing tubes to cool to room temperature, $1.0 \mathrm{~mL}$ of $\mathrm{BF}_{3}, 14 \%$ in methanol (Sigma-Aldrich, Sweden), was added to the samples and the incubation repeated. After allowing tubes to cool to room temperature, $0.4 \mathrm{~mL}$ of milli-Q water and $0.4 \mathrm{~mL}$ of hexane were added; tubes were vortexed and then centrifuged $(2,500 \times g$ for $6 \mathrm{~min})$. The organic layer containing the fattyacid methyl-esters (FAMEs) was transferred to a new tube and prepared for GC-MS as described in "Analysis of fatty acids by GC-MS" section.

\section{In-house two-step transesterification with $\mathrm{KOH}$}

To the microalgae, $4.0 \mathrm{~mL}$ of ethanol-methanol (ratio $3: 2 v / v$ ) was added, containing $0.05 \%(w / v)$ of the antioxidant BHT. Potassium hydroxide $(\mathrm{KOH})$ pellets, $0.4 \mathrm{~g}$, were added and the tubes shaken until the pellets had dissolved (roughly $20 \mathrm{~min}$ ). Tubes were incubated in a heating block at $70{ }^{\circ} \mathrm{C}$ for $120 \mathrm{~min}$, with manual shaking every $10 \mathrm{~min}$. After cooling the tubes to room temperature, $5.0 \mathrm{~mL}$ of toluene were added, and the tubes were vortexed briefly. To induce a two-phase system and protonate the fatty acids, $2.0 \mathrm{~mL}$ of $6 \mathrm{M} \mathrm{HCl}$ was added. Tubes were centrifuged at 2,000 $\times g$ for $6 \mathrm{~min}$, and the organic phase was transferred to a fresh tube and methylated as described in "In-house methanolic- $\mathrm{HCl}$ transesterification" 
section and separated as described in "Analysis of fatty acids by GC-MS" section.

Direct-transesterification methods (D-TE)

\section{Griffiths methanolic- $B F_{3}$ transesterification}

This method is a shortened version of Griffiths et al.'s method ("Griffiths et al.'s transesterification" section), omitting the incubation with sodium methoxide: Dry samples were incubated with $1.0 \mathrm{~mL}$ of $\mathrm{BF}_{3}$ at $80^{\circ} \mathrm{C}$ and FAMEs extracted into hexane. FAMEs were prepared for GC-MS as described in "Analysis of fatty acids by GC-MS" section.

\section{Christie's methanolic- $\mathrm{H}_{2} \mathrm{SO}_{4}$ transesterification}

This method is a scaled-down version of that previously published by Christie [26]. To $20 \mathrm{mg}$ algae, $0.5 \mathrm{~mL}$ toluene and $1.0 \mathrm{~mL}$ of $1 \%(v / v) \mathrm{H}_{2} \mathrm{SO}_{4}$ in methanol were added. Tubes were flushed with nitrogen gas under manual agitation for $10 \mathrm{~s}$ before capping. Capped tubes were incubated at $50{ }^{\circ} \mathrm{C}$ for $17 \mathrm{~h}$. Thereafter, $5.0 \mathrm{~mL}$ of $5 \%(w / v)$ aqueous $\mathrm{NaCl}$ were added; tubes were vortexed briefly, and $5.0 \mathrm{~mL}$ of hexane was added. After vortexing, phases were separated by centrifuging $(2,500 \times g, 6 \mathrm{~min})$. The hexane phase was recovered, and the residue was re-extracted with a fresh aliquot of hexane. The two hexane phases were pooled. A small amount of the pooled hexane phase was extracted with water and the water's $\mathrm{pH}$ tested on indicator paper (Macherey-Nagel Tri-test, Germany). Since the $\mathrm{pH}$ of the water phase was 6-7, the following steps described in the original method were omitted: The washing step with aqueous bicarbonate and subsequent drying over sodium sulfate. GC-MS was performed as described in "Analysis of fatty acids by GC-MS" section.

\section{Lewis et al.'s methanolic-HCl transesterification}

This method was first published by Lewis et al. and used by Burja et al. in their comparison of methods [23, 34]. Briefly, microalgae were incubated at $90{ }^{\circ} \mathrm{C}$ for 120 min with methanol-HCl-chloroform (10:1:1). Next, $1.0 \mathrm{~mL}$ of milli-Q water was added and the FAMEs extracted by adding $2.0 \mathrm{~mL}$ hexane-chloroform $(4: 1)$, vortexing and recovering the top layer, repeating the extraction a total of three times. The pooled organic layer was prepared for GC-MS, as described in "Analysis of fatty acids by GC-MS" section.

\section{In-house methanolic-HCl transesterification}

This in-house method is loosely based on that of Lepage and Roy [35]. This method was used both to directly methylate fatty acids in algae and to methylate fatty acid extracts from the chloroform-methanol methods and the in-house 2-TE. For direct methylation, $1.0 \mathrm{~mL}$ of toluene was added to the microalgae. For the other samples, i.e. methylation of extracts, solvent was first evaporated under a stream of nitrogen at $40{ }^{\circ} \mathrm{C}$, and residues were re-suspended in $1.0 \mathrm{~mL}$ of toluene. Once toluene had been added, the procedure was identical for all samples: The $1.0 \mathrm{~mL}$ of freshly prepared $10 \%(\mathrm{v} / \mathrm{v})$ acetyl chloride in methanol was added, and tubes were incubated for $120 \mathrm{~min}$ at $70^{\circ} \mathrm{C}$. Tubes were allowed to cool, and the reaction was terminated by adding $0.2 \mathrm{~mL}$ of milli-Q water. FAMEs were extracted by adding $5.0 \mathrm{~mL}$ of petroleum ether-diethyl ether (4:1), vortexing briefly, centrifuging $(2,500 \times g$ for $6 \mathrm{~min})$ and transferring the organic (upper) phase to a fresh tube. GCMS was performed as described in "Analysis of fatty acids by GC-MS" section.

\section{In-house methanolic-HCl transesterification with overnight incubation}

This method was applied only for direct-methylation purposes. The method is identical to the above ("In-house methanolic- $\mathrm{HCl}$ transesterification"), with the exception that the incubation at $70{ }^{\circ} \mathrm{C}$ for $120 \mathrm{~min}$ was exchanged for incubation in the dark at room temperature and shaking at $300 \mathrm{rpm}$ for $24 \mathrm{~h}$.

\section{Analysis of fatty acids by GC-MS}

Solvent from extracts was evaporated under a stream of nitrogen at $40{ }^{\circ} \mathrm{C}$ and FAMEs re-suspended in 2,2,4trimethylpentane. The extract was injected into an Agilent 7890 A GC system equipped with a J\&W DB-wax column $(30 \mathrm{~m} \times 0.250 \mathrm{~mm} \times 0.25 \mu \mathrm{m})$ and interfaced with a Agilent 5975 C triple-axis mass spectrometric (MS) detector in electron impact mode. Injection volume was $1 \mu \mathrm{L}$ with a $15: 1$ split at an inlet temperature of $275^{\circ} \mathrm{C}$. The carrier gas was helium, with a fixed flow of $1 \mathrm{~mL} / \mathrm{min}$ throughout the temperature program, which was as following $-100^{\circ} \mathrm{C}$ for $0 \mathrm{~min}$, ramp at $4{ }^{\circ} \mathrm{C} / \mathrm{min}$ to $205^{\circ} \mathrm{C}$, thereafter ramp at $1{ }^{\circ} \mathrm{C} / \mathrm{min}$ to $230{ }^{\circ} \mathrm{C}$, hold $5 \mathrm{~min}$. Three external standards of FAME mixtures were used for identification of the different peaks in algal samples: GLC 463 (Nu-Chek prep, Inc., Elysian, USA), PUFA-3 (Supelco, Bellefonte, USA), C22:5 n-6 (Larodan, Malmö, Sweden). Fatty acids were quantified against the internal standard, summed, and expressed as milligrams FA per gram dry algae biomass.

\section{Statistical analysis}

To investigate statistical differences between the means of different individual methods, Kruskal-Wallis test was applied, followed by Kruskal-Wallis stepwise step-down. KruskalWallis is a non-parametric test and was used because the small number of replicates ( $n=3$, in most cases) did not allow to 
check if measurements were normally distributed. However, we expect a normal distribution and therefore report means and standard deviation. The software used was SPSS 19 (IBM). Means were considered significantly different when $p \leq 0.05$.

\section{Results}

Comparison of chloroform-methanol extractions, 2-TE, and D-TE methods

The chloroform-methanol-based extractions recovered significantly less total fatty acids than either the 2-TE or D-TE methods in $N$. oculata and P. tricornutum. The 2-TE methods and D-TE methods recovered approximately the same amounts of fatty acids from the same material. In I. galbana, the range of results was quite small (see Fig. 2a) with Lee et al.'s method recovering $117 \mathrm{mg} \mathrm{FA} / \mathrm{g}$ dry algae and D-TE with either $\mathrm{H}_{2} \mathrm{SO}_{4}$ or $\mathrm{HCl}$ recovering $126 \mathrm{mg} / \mathrm{g}$. Since all methods roughly agree, these results indicate that I. galbana is simple to analyze, and many methods therefore are valid. However, in $N$. oculata, there was a pronounced difference between chloroform-methanol extractions and the two groups of transesterification methods (see Fig. 2b), where the acidic Bligh and Dyer recovered as little as $83 \mathrm{mg} / \mathrm{g}$, while Griffith et al.'s 2-TE and the in-house $\mathrm{HCl}$ D-TE recovered $126 \mathrm{mg} / \mathrm{g}$. For some algae, obviously, the method of fatty acid extraction has a significant impact on the final results. In P. tricornutum (Fig. 2c), the chloroform-methanol-based extractions gave widely varying results. Although the Bligh and Dyer method appears to have recovered more fatty acids than any other method, this is an error caused by insufficient extraction of the internal standard (see "Acidification of Bligh and Dyer" section). Although increasing the extraction time of the acidified Bligh and Dyer to $24 \mathrm{~h}$ in P. tricornutum did lead to yields approaching that of the transesterification methods in this particular alga, the transesterification methods give a narrower range of results in all three tested microalgae. The differences between Bligh and Dyer's and Lee et al.'s method on one side and the transesterification methods on the other was significant for both $N$. oculata and P. tricornutum.

For all three types of algae, the two 2-TE methods (Griffiths et al.'s method and the in-house $\mathrm{KOH}$ method) did not differ significantly in total FAMEs recovered. The 2-TE methods recovered approximately as much fatty acids as the D-TE methods. Although some significant differences were noted, no obvious trend emerged. For I. galbana (Fig. 2a), the results for the 2-TE methods were on the low end of the range for transesterification results while, for $N$. oculata (Fig. 2b), the 2-TE methods were on the high end of the transesterification results. For $P$. tricornutum, no strong differences between the 2-TE methods and D-TE methods emerged.

The D-TE methods recovered high amounts of FAMEs in all three microalgae. Amongst the D-TE methods, the $\mathrm{BF}_{3}-$ method recovered significantly less fatty acids than any of the other D-TE methods. For all three species of microalgae, the methods of Christie, Lewis et al., and the in-house $\mathrm{HCl}$ method at $70{ }^{\circ} \mathrm{C}$ recovered among the most FAMEs. The inhouse $\mathrm{HCl}$-method at room temperature recovered significantly less total FAMEs than Christie's method and Lewis et al.'s method in I. galbana.

\section{Acidification of Bligh and Dyer}

After performing the Bligh and Dyer on P. tricornutum, it was suspected that the internal standard (C23:0) had not been fully extracted (see Fig. 2c), based on the standard's small area in the chromatogram (data not shown), resulting in an unanticipated high recovery of FAMEs. Both for $N$. oculata and P. tricornutum (Fig. 2b and c), the total apparent FAMEs decreased when using diluted acid instead of milli-Q water to break the monophasic system. The $\mathrm{pH}$ of water suspensions of the various microalgae was as follows: I. galbana $\mathrm{pH} 6, \mathrm{~N}$. oculata $\mathrm{pH} 7$, and P. tricornutum $\mathrm{pH}$ 9; the $\mathrm{pH}$ of the aqueous phase remaining after the acidified extraction was $\mathrm{pH} 2,3$, and 5 , respectively. Since the $\mathrm{pH}$ is expected to stay the same in the non-acidified Bligh and Dyer, the results indicate that the high $\mathrm{pH}$ in $P$. tricornutum and $N$. oculata samples caused the internal standard to be incompletely extracted, thereby overestimating the true FAME-content of the sample. It can be expected that other fatty acids present in the sample were also only partially extracted.

Fatty acid profiles

Fatty acid profiles (see Electronic Supplementary Material, Tables S1, S2, and S3) for the various methods were compared, to explore if methods showed preference for certain fatty acids. Though the percentage of many fatty acids was significantly different, no obvious pattern emerged. Instead of the entire patterns as a measure for preferential extraction of certain fatty acids, saturated fatty acids (SFAs), monounsaturated fatty acids (MUFAs), and polyunsaturated fatty acids (PUFAs) were summed and expressed as percentage of total extracted fatty acids (see Fig. 3). Although differences between different methods generally are quite small, the chloroform-methanol-based extractions recovered a significantly lower percentage of SFAs than the 2-TE and D-TE methods. In I. galbana and N. oculata, the decrease in SFA percentage in chloroform-methanol methods was offset by an increase in MUFA. In P. tricornutum, the decrease in SFA percentage is the most visible (Fig. 3c) and is offset by an increase in PUFA percentage. 
Fig. 2 Total fatty acid content of three different algae, $\mathbf{A}$

I. galbana, $\mathbf{B}$ N. oculata, $\mathbf{C}$

P. tricornutum, as determinded by

11 different methods: solid bars

represent chloroform-methanol

extractions ("B\&D"=Bligh and

Dyer; acidified Bligh and Dyer;

acidified Bligh and Dyer,

overnight; Lee et al.), cross-

hatched bars represent two-step

transesterification (Griffiths

et al.'s full method; in-house

$\mathrm{KOH}$ method), and speckled bars

represent direct-transesterification

(short version of Griffiths et al.'

method with boron trifluoride;

Christie's method; Lewis et al.'s

method; "HCl" =in-house acetyl

chloride in methanol; in-house

acetyl chloride in methanol,

overnight). Error bars show \pm

standard deviation, $n=3$ for all but

$\mathrm{HCl}$ where $n=12$. The high value

for the Bligh and Dyer (leftmost

bar) in $\mathbf{C}$ is an artifact caused by inadequate extraction of the in-

ternal standard
A

$\square \mathrm{CHCl} 3-$

$\mathrm{MeOH}$

2-TE

口 D-TE

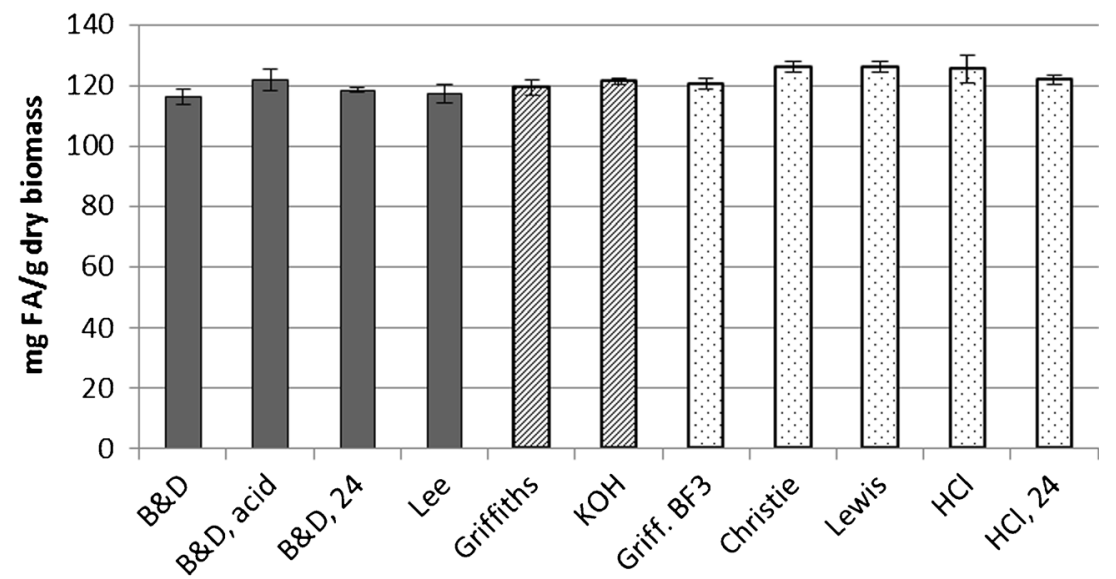

B

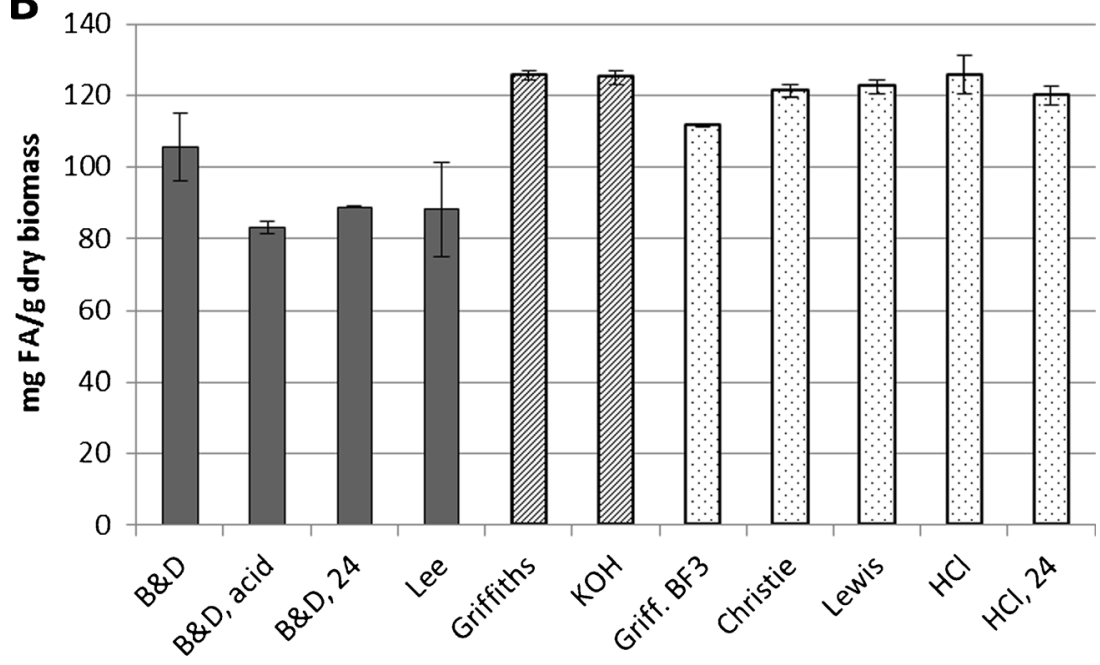

C

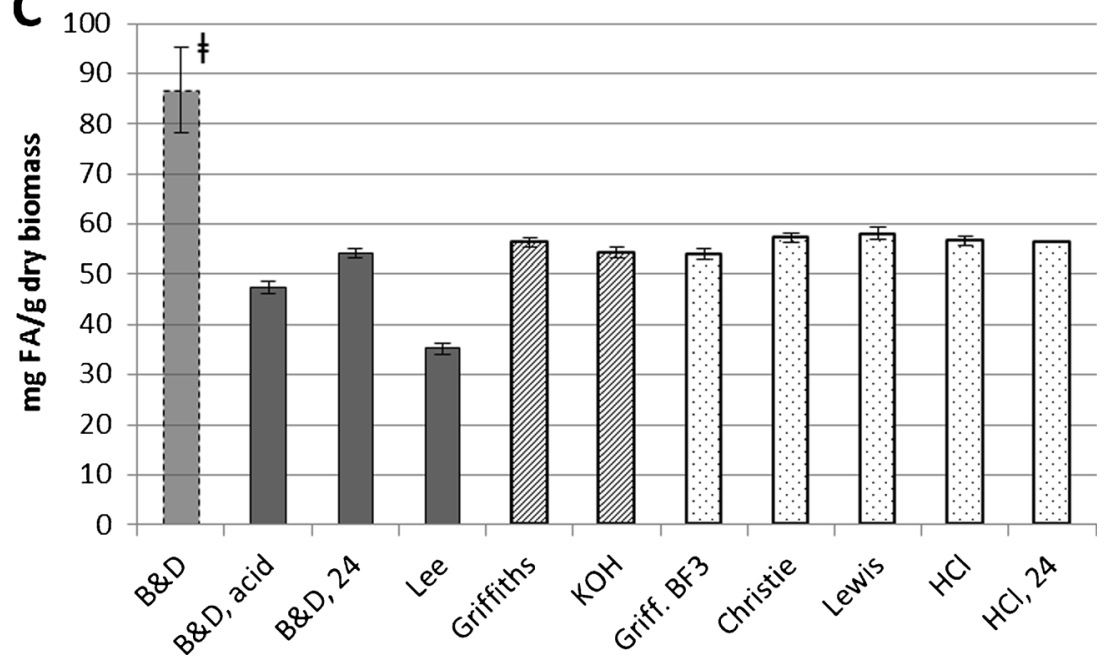




\section{Discussion}

Our results indicate that three D-TE methods (Christie's method, Lewis et al.'s method, and the in-house $\mathrm{HCl}$ method) gave identical measures of total fatty acids on I. galbana, $N$. oculata, and P. tricornutum. Furthermore, two 2-TE methods (Griffiths et al.'s, in-house $\mathrm{KOH}$ ) and the D-TE inhouse overnight $\mathrm{HCl}$-method gave results close to the three aforementioned methods. Methods based on chloroformmethanol-extraction recovered as much fatty acids as the 2TE and D-TE methods for I. galbana. However, chloroformmethanol-extractions did not succeed in recovering as much fatty acids from $N$. oculata and P. tricornutum; this was expected, considering that (1) the algae had been chosen for their differences in cell wall composition and (2) other groups report $N$. oculata to be difficult to extract [36]. These results are in agreement with those of Griffiths et al., who compared various chloroform-methanol extractions to a 2-TE method on three microalgae including Nannochloropsis sp. and found the 2-TE method to consistently recover more fatty acids [28]. However, our results do not agree with Burja et al.'s study in which a Bligh and Dyer-based method recovered most oil from Thraustochytrium sp., closely followed by two different 2-TE methods, while Lewis et al.'s D-TE method recovered much less [23]. In our study, Lewis et al.'s method repeatedly gave high results, in contradiction to Burja et al.'s study. A possible explanation for the different results is that we quantified FAMEs from the Bligh and Dyer extraction by GC-MS instead of determining total lipids gravimetrically. While GCMS allows quantification of specific molecules, gravimetric lipid determination may include varying amounts of hydrophobic and semi-hydrophobic impurities, depending on the solvent used to extract the lipids.

Lee et al.'s method, which was developed for fatty fish $(>6 \%)$ and uses a higher ratio of chloroform to methanol (2:1), did not recover more fatty acids than the original Bligh and Dyer method, even though the algae contained $>5 \%$ fatty acids. Lee et al. suggest themselves that the method is applicable to fish in which the fatty acids are mainly bound to triacylglycerols [19]. In the present study, the lipid classes were not assessed, i.e., the compounds to which the fatty acids were esterified were not determined. However, Ryckebosch et al. recently measured the lipid classes of Isochrysis, Nannochloropsis, and Phaeodactylum, showing that at least $40 \%$ of the lipids extracted by their Bligh and Dyer method were phospholipids and glycolipids [37]. Our results also agree with Ryckebosch et al.'s earlier work where different solvent systems were compared on Chlorella, and the authors showed that a 1:1 ratio of chloroform-methanol recovered significantly more total lipids than a 2:1 ration of chloroform-methanol.

A further caveat of the chloroform-methanol extractions was apparent in P. tricornutum, where SFAs were recovered to a lesser degree than in the transesterification methods, indicating that the chloroform-methanol methods may be biased against SFAs. On closer inspection, the SFA C16:0 was recovered to a lesser extent. Other authors studying microalgae have found $\mathrm{C} 16: 0$ to be present to the greatest extent in three lipids classes: (1) sulfoquinovosyldiacylglycerol [38, 39], (2) triacylglycerol [38], and (3) an unidentified phosopholipid [39]. Based on Ryckebosch et al.'s work [37], it seems likely that the chloroform-methanol extraction efficiently recovers the polar lipids-glycolipids and phospholipids-from P. tricornutum but recovers less of the more non-polar triacylglycerol-fraction. The recovery of all lipid classes by the various methods should be investigated in future work by adding, e.g., triacylglycerol, phospholipid, glycolipid, and fatty acid internal-standards to ensure that the methods are not biased towards any specific lipid class, such as fatty acid, used in this study.

In the Bligh and Dyer extraction, we noted the necessity to extract under acidic conditions in order to recover as much of the sample's fatty acids as possible, which, in our case, was mainly the added internal standard. The presence of acid is expected to improve the extraction of free fatty acids for two reasons: (1) fatty acids become protonated, shifting their partitioning coefficient in favor of the organic phase and (2) the aqueous phase becomes more polar in the presence of ionic species (here: chloride and hydronium ions), resulting in non-polar molecules (such as lipids) being excluded to a greater degree, thus, in effect, shifting the partitioning coefficient of lipids in favor of the non-polar phase. It could be argued that acidic conditions should have been applied to Lee et al.'s extraction, but the area of the internal-standard in the chromatogram was similar to that of 2-TE and D-TE methods, indicating that the internal-standard had been recovered to the same extent; presumably, the addition of $0.5 \%$ salt to the aqueous-phase was sufficient to make the aqueous-phase polar enough to recover the fatty acids.

The 2-TE methods gave high yields and small variations, yet it is difficult to justify the use of expensive chemicals such as sodium methoxide or long extraction times and many manipulations (e.g., requiring a full day's lab work for 12 samples for the in-house KOH-method) when the D-TE methods give equally good results. At the onset of this study, we believed that more complete cell disruption would facilitate the extraction of cellular components; however, the inhouse 2-TE method with $\mathrm{KOH}$, specifically designed to achieve this, did not result in higher yield in all microalgae. Though the $\mathrm{KOH}$ method uses cheap reagents, it requires far more manipulation and time.

Among the D-TE methods, all except the $\mathrm{BF}_{3}$-method appeared to be equivalent in respect to the amount of total FAMEs recovered. Although the $\mathrm{BF}_{3}$-method recovered significantly less FAMEs in N. oculata and P. tricornutum, this is not necessarily caused by the catalyst, but rather by the short 
Fig. 3 Saturated (grey, bottom), monounsaturated (light grey, middle), and polyunsaturated fatty acids (medium grey, top) as determined by different methods, in A I. galbana, B N. oculata, C P. tricornutum. Fatty acid groups are expressed as percent of total fatty acids. Error bars indicate \pm standard deviation; $n=3$ except for $\mathrm{HCl}$, where $n=12$. For method abbreviations, refer to Fig. 2
A

$\square$ SFA $\square$ MUFA $\square$ PUFA
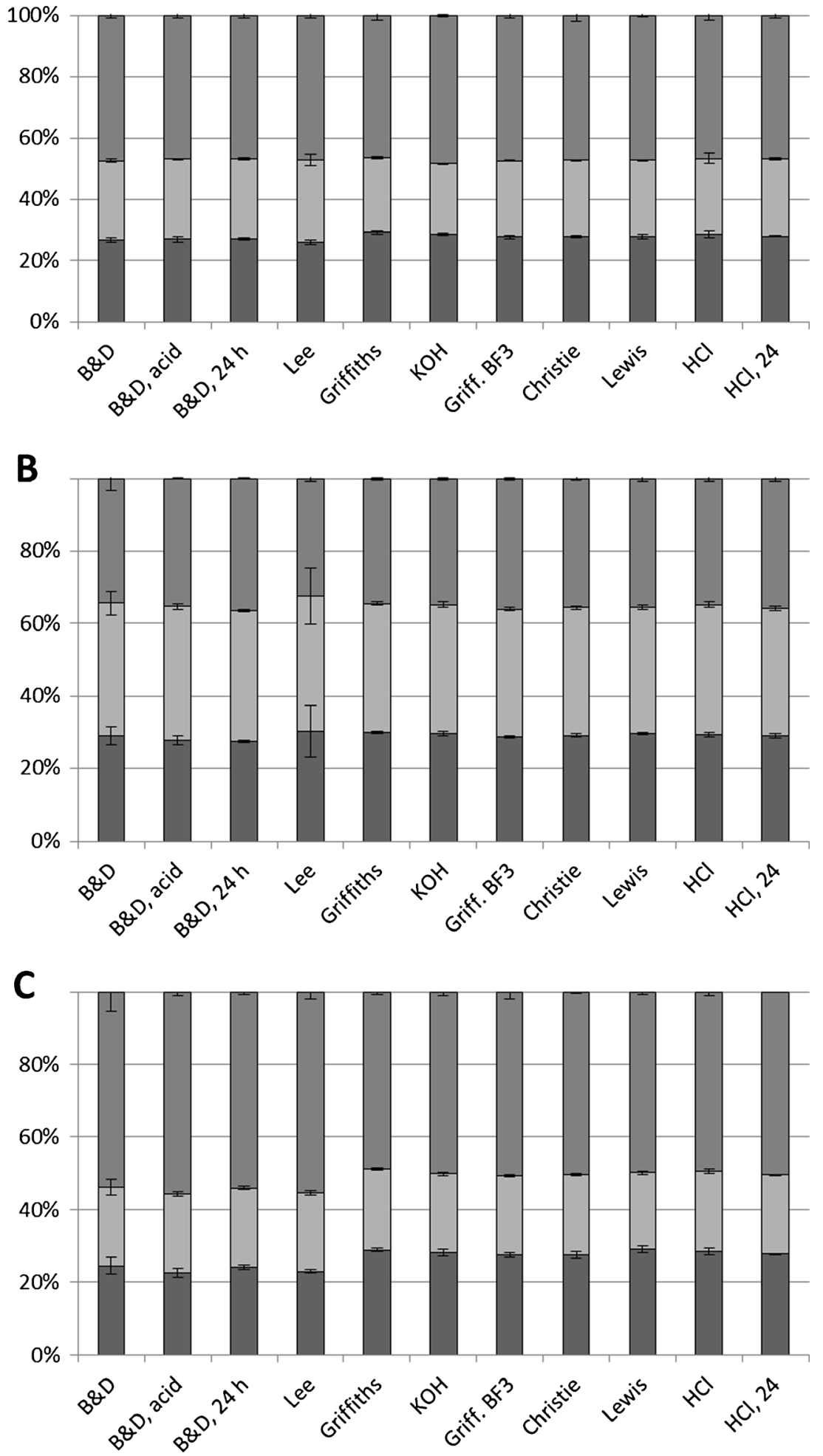

incubation time compared with other D-TE methods. It is possible that increasing the incubation time would improve the FAME-recovery for the $\mathrm{BF}_{3}$-method; however, we decided against proceeding with this catalyst, since it is hazardous to work with, expensive, and its shelf life is limited [20]. Since Christie's D-TE with $\mathrm{H}_{2} \mathrm{SO}_{4}$, Lewis et al.'s method with $\mathrm{HCl}$, and the in-house $\mathrm{HCl}$-method all gave similar results, we would recommend any of these three for determination of 
FAMEs in microalgae. While the $\mathrm{H}_{2} \mathrm{SO}_{4}$-reagent is easy to prepare, the incubation takes longer than the other two.

The method developed by Lewis et al. uses methanolic$\mathrm{HCl}$ with chloroform, a reagent which is easier to prepare than mixing methanol and acetyl chloride. The problem of chloroform sinking is elegantly circumvented by extracting the FAMEs into hexane. However, for those who wish to avoid chlorinated solvents, our in-house D-TE method comprising $10 \%$ acetyl chloride in methanol presents an alternative. Acetyl chloride is less toxic than, e.g., boron trifluoride, though care must be taken when preparing the methylation reagent, since acetyl chloride reacts violently with methanol [26]. Although acetyl chloride is a chlorinated solvent in itself, the chlorine is ejected in reactions with water and alcohol, rendering less toxic products. Furthermore, we provide two versions of the in-house method which, here, gave similar results in the tested microalgae: Incubation can take place either at $70{ }^{\circ} \mathrm{C}$ for $120 \mathrm{~min}$ or at room temperature overnight. In both versions, it may be possible to shorten the incubation time, a possible focus for future work.

In conclusion, if the aim is to quantify total fatty acids from microalgae, the D-TE methods are faster and require less handling than do the 2-TE methods and the chloroformmethanol-based extractions, although results are generally similar. The D-TE methods utilizing hydrochloric acid or sulfuric acid catalysts recovered the most fatty acids of all methods here compared, with the added benefit that these DTE methods do not necessarily require halogenated solvents. Lee et al.'s chloroform - methanol extraction recovered less fatty acids than any of the other methods in $N$. oculata and P. tricornutum, but, for I. galbana, all tested methods recovered roughly the same amount of fatty acids, indicating a significant matrix effect for different microalgae.

Acknowledgments We thank Chalmers University of Technology, Department of Chemical and Biological Engineering, for funding of this project, and Kristina Liljestrand, Karin Larsson, Cecilia Tullberg, and Hanna Harrysson for their assistance in preparing this manuscript.

Open Access This article is distributed under the terms of the Creative Commons Attribution License which permits any use, distribution, and reproduction in any medium, provided the original author(s) and the source are credited.

\section{References}

1. Bligh EG, Dyer WJ (1959) A rapid method of total lipid extraction and purification. Can J Biochem Physiol 37(8):911-917

2. Axelsson M, Gentili F (2014) A single-step method for rapid extraction of total lipids from green microalgae. PLoS One 9(2). doi:10. 1371/journal.pone.0089643

3. Carvalho AP, Malcata FX (2005) Optimization of omega-3 fatty acid production by microalgae: crossover effects of $\mathrm{CO} 2$ and light intensity under batch and continuous cultivation modes. Mar Biotechnol 7(4):381-388. doi:10.1007/s10126-004-4047-4

4. Cohen Z, Khozin-Goldberg I, Adlerstein D, Bigogno C (2000) The role of triacylglycerol as a reservoir of polyunsaturated fatty acids for the rapid production of chloroplastic lipids in certain microalgae. Biochem Soc Trans 28:740-743. doi:10.1042/0300-5127:0280740

5. Fábregas J, Maseda A, Domínguez A, Otero A (2004) The cell composition of Nannochloropsis sp. changes under different irradiances in semicontinuous culture. World J Microbiol Biotechnol 20(1):31-35. doi:10.1023/B:WIBI.0000013288.67536.ed

6. Graziani G, Schiavo S, Nicolai MA, Buono S, Fogliano V, Pinto G, Pollio A (2013) Microalgae as human food: chemical and nutritional characteristics of the thermo-acidophilic microalga Galdieria sulphuraria. Food Funct 4(1):144-152. doi:10.1039/c2fo30198a

7. Halim R, Gladman B, Danquah MK, Webley PA (2011) Oil extraction from microalgae for biodiesel production. Bioresour Technol 102(1):178-185. doi:10.1016/j.biortech.2010.06.136

8. Klok AJ, Martens DE, Wijffels RH, Lamers PP (2013) Simultaneous growth and neutral lipid accumulation in microalgae. Bioresour Technol 134:233-243

9. Mansour MP (2005) Reversed-phase high-performance liquid chromatography purification of methyl esters of C16-C28 polyunsaturated fatty acids in microalgae, including octacosaoctaenoic acid [28: 8(n-3)]. J Chromatogr A 1097(1-2):54-58

10. Ohta S, Chang T, Aozasa O, Kondo M, Miyata H (1992) Sustained production of arachidonic and eicosapentaenoic acids by the red algae Porphyridium purpureum cultured in a light/dark cycle. J Ferment Bioeng 74(6):398-402. doi:10.1016/0922-338x(92)900402

11. Patil V, Kallqvist T, Olsen E, Vogt G, Gislerod HR (2007) Fatty acid composition of 12 microalgae for possible use in aquaculture feed. Aquac Int 15(1):1-9. doi:10.1007/s10499-006-9060-3

12. Ranjan A, Patil C, Moholkar VS (2010) Mechanistic assessment of microalgal lipid extraction. Ind Eng Chem Res 49(6):2979-2985. doi:10.1021/ie9016557

13. Ǩezanka T, Lukavský J, Nedbalová L, Kolouchová I, Sigler K (2012) Effect of starvation on the distribution of positional isomers and enantiomers of triacylglycerol in the diatom Phaeodactylum tricornutum. Phytochemistry 80:17-27. doi:10.1016/j.phytochem. 2012.05.021

14. Sheng J, Vannela R, Rittrnann BE (2011) Evaluation of methods to extract and quantify lipids from Synechocystis PCC 6803. Bioresour Technol 102(2):1697-1703. doi:10.1016/j.biortech.2010.08.007

15. Sukenik A, Wahnon R (1991) Biochemical quality of marine unicellular algar with special emphasis on lipid composition I. Isochrysis galbana. Aquaculture 97:61-72

16. Vazhappilly R, Chen F (1998) Heterotrophic production potential of omega-3 polyunsaturated fatty acids by microalgae and algae-like microorganisms. Bot Mar 41(6):553-558

17. Xu X-Q, Beardall J (1997) Effect of salinity on fatty acid composition of a green microalga from an antarctic hypersaline lake. Phytochemistry 45(4):655-658. doi:10.1016/s0031-9422(96)00868-0

18. Yongmanitchai W, Ward OP (1991) Screening of algae for potential alternative sources of eicosapentaenoic acid. Phytochemistry 30(9): 2963-2967. doi:10.1016/s0031-9422(00)98231-1

19. Lee CM, Trevino B, Chaiyawat M (1996) A simple and rapid solvent extraction method for determining total lipids in fish tissue. J AOAC Int 79(2):487-492

20. Liu KS (1994) Preparation of fatty acid methyl esters for gaschromatographic analysis of lipids in biological materials. J Am Oil Chem Soc 71(11):1179-1187

21. Galbe M, Zacchi G (2012) Pretreatment: the key to efficient utilization of lignocellulosic materials. Biomass Bioenerg 46:70-78. doi: 10.1016/j.biombioe.2012.03.026

22. Cartens M, Grima EM, Medina AR, Gimenez AC, Gonzalez JI (1996) Eicosapentaenoic acid (20:5n-3) from the marine microalga 
Phaeodactylum tricornutum. J Am Oil Chem Soc 73(8):1025-1031. doi:10.1007/bf02523411

23. Burja AM, Armenta RE, Radianingtyas H, Barrow CJ (2007) Evaluation of fatty acid extraction methods for Thraustochytrium sp ONC-T18. J Agric Food Chem 55(12):4795-4801. doi:10.1021/ jf070412s

24. Sukenik A (1991) Ecophysiological considerations in the optimization of eicosapentaenoic acid production by nannochloropsis-Sp (Eustigmatophyceae). Bioresour Technol 35(3):263-269

25. Carrapiso AI, Garcia C (2000) Development in lipid analysis: some new extraction techniques and in situ transesterification. Lipids 35(11):1167-1177

26. Christie WW (1989) Analysis of fatty acids. Gas chromatography and lipids. P.J. Barnes \& Associates (The Oily Press Ltd), Dundee

27. Ryckebosch E, Muylaert K, Foubert I (2012) Optimization of an analytical procedure for extraction of lipids from microalgae. J Am Oil Chem Soc 89(2):189-198. doi:10.1007/s11746-011-1903-z

28. Griffiths MJ, Van Hille RP, Harrison STL (2010) Selection of direct transesterification as the preferred method for assay of fatty acid content of microalgae. Lipids 45(11):1053-1060

29. Borowitzka MA, Volcani BE (1978) The polymorphic diatom Phaeodactylum tricornutum: ultrastructure of its morphotypes. J Phycol 14(1):10-21. doi:10.1111/j.1529-8817.1978.tb00625.x

30. De Martino A, Meichenin A, Shi J, Pan KH, Bowler C (2007) Genetic and phenotypic characterization of Phaeodactylum tricornutum (Bacillariophyceae) accessions. J Phycol 43(5):9921009. doi:10.1111/j.1529-8817.2007.00384.x

31. Sari YW, Bruins ME, Sanders JPM (2013) Enzyme assisted protein extraction from rapeseed, soybean, and microalgae meals. Ind Crop Prod 43:78-83. doi:10.1016/j.indcrop.2012.07.014
32. Gelin F, Boogers I, Noordeloos AAM, Sinninghe Damsté JS, Riegman R, De Leeuw JW (1997) Resistant biomacromolecules in marine microalgae of the classes Eustigmatophyceae and Chlorophyceae: geochemical implications. Org Geochem 26(1112):659-675. doi:10.1016/S0146-6380(97)00035-1

33. Payne MF, Rippingale RJ (2000) Evaluation of diets for culture of the calanoid copepod Gladioferens imparipes. Aquaculture 187(1-2): 85-96. doi:10.1016/S0044-8486(99)00391-9

34. Lewis T, Nichols PD, McMeekin TA (2000) Evaluation of extraction methods for recovery of fatty acids from lipid-producing microheterotrophs. J Microbiol Methods 43(2):107-116. doi:10. 1016/s0167-7012(00)00217-7

35. Lepage G, Roy CC (1986) Direct transesterification of all classes of lipids in a one-step reaction. J Lipid Res 27(1):114-120

36. Slocombe SP, Zhang Q, Black KD, Day JG, Stanley MS (2012) Comparison of screening methods for high-throughput determination of oil yields in micro-algal biofuel strains. J Appl Phycol:1-12

37. Ryckebosch E, Bruneel C, Termote-Verhalle R, Muylaert K, Foubert I (2014) Influence of extraction solvent system on extractability of lipid components from different microalgae species. Algal Res 3(1): 36-43. doi:10.1016/j.algal.2013.11.001

38. Yongmanitchai W, Ward OP (1992) Separation of lipid classes from Phaeodactylum tricornutum using silica cartridges. Phytochemistry 31(10):3405-3408. doi:10.1016/0031-9422(92)83694-t

39. Alonso DL, Belarbi EH, Fernandez-Sevilla JM, Rodriguez-Ruiz J, Grima EM (2000) Acyl lipid composition variation related to culture age and nitrogen concentration in continuous culture of the microalga Phaeodactylum tricornutum. Phytochemistry 54(5):461-471. doi:10. 1016/s0031-9422(00)00084-4 